\title{
Possibility of AnteOwl IVUS-based antegrade dissection and reentry using the tip detection method for CTO-PCI
}

\author{
Atsunori Okamura ${ }^{1} \cdot$ Hiroyuki Nagai ${ }^{-} \cdot$ Kota Tanaka $^{1} \cdot$ Satoshi Suzuki $^{1} \cdot$ Heitaro Watanabe $^{1} \cdot$ Katsuomi Iwakura $^{1}$
}

Received: 4 January 2022 / Accepted: 6 February 2022 / Published online: 15 February 2022

(c) The Author(s) 2022

Keywords Coronary intervention · Chronic total occlusion · IVUS-based ADR · Tip detection method

\author{
Abbreviations \\ ADR Antegrade dissection and reentry \\ AO-IVUS AnteOwl WR-intravascular ultrasound \\ CTO Chronic total occlusion \\ IVUS Intravascular ultrasound
}

In chronic total occlusion (CTO) intervention, it has been determined that it is impossible to create a reentry from a subintima to a true lumen with regular guidewire manipulation. Therefore, antegrade dissection and reentry (ADR) using Stingray system (Boston Scientific, Natick, MA, USA) was developed. In the present case, ADR using a Stingray balloon failed to create a reentry. Intravascular ultrasound (IVUS) observation revealed the damaged part of the wall by ADR. We could create a reentry at the damaged part by an exact vertical directional puncture using the tip detection method with an AnteOwl WR-IVUS (AO-IVUS; Terumo Corp., Tokyo, Japan) [1].

A 74-year-old man suffered from effort angina pectoris due to a CTO lesion in the left anterior descending coronary artery because of occlusion of bypass grafting to it. Computed tomography and angiography showed severe calcification in the CTO (Fig. 1A, B). An 8 Fr guide catheter was inserted from the femoral artery. Any guidewires including a Confianza-20 g (Asahi Intecc Co., Ltd., Aichi,
Japan) could not enter the severe calcified lesion, but a XT-A wire (Asahi Intecc) supported by over-the-wire balloon anchoring could be advanced through the subintimal space using the knuckle wiring. We moved on to angiography-based ADR using a Stingray balloon. Using Confianza-20 g and XT-R wires, five attempts of the stick-andswap technique were performed, but the guidewire could not be led into the true lumen which was not visible by angiography. AO-IVUS observation revealed the somewhat damaged part of the true lumen wall which might have been caused by the punctures during ADR $6 \mathrm{~mm}$ beyond the CTO exit (Fig. 1E). A vertical puncture might not be possible by angiography-based ADR because it depends on fluoroscopy. We moved onto AO-IVUS-based ADR using the tip detection method to perform an exact vertical directional puncture at the damaged part (Fig. 1C). A GAIA Next 3 supported by a Corsair (Asahi Intecc) was used because penetration force would be required for reentry. The tip detection method allowed the tip of the wire to puncture the wall of the true lumen in an exactly vertical direction, resulting in the successful reentry (Fig. 1F-H, ESM Video 1). Normal antegrade blood flow was achieved after stenting (Fig. 1D). Going forward, if guidewires with more penetrating force are used, they may allow for intentional reentry even for the undamaged walls using the AOIVUS-based tip detection method.
Atsunori Okamura

a_okamura@watanabe-hsp.or.jp

1 Cardiovascular Center, Division of Cardiology, Sakurabashi Watanabe Hospital, 2-4-32 Umeda, Kita-ku, Osaka 530-0001, Japan 

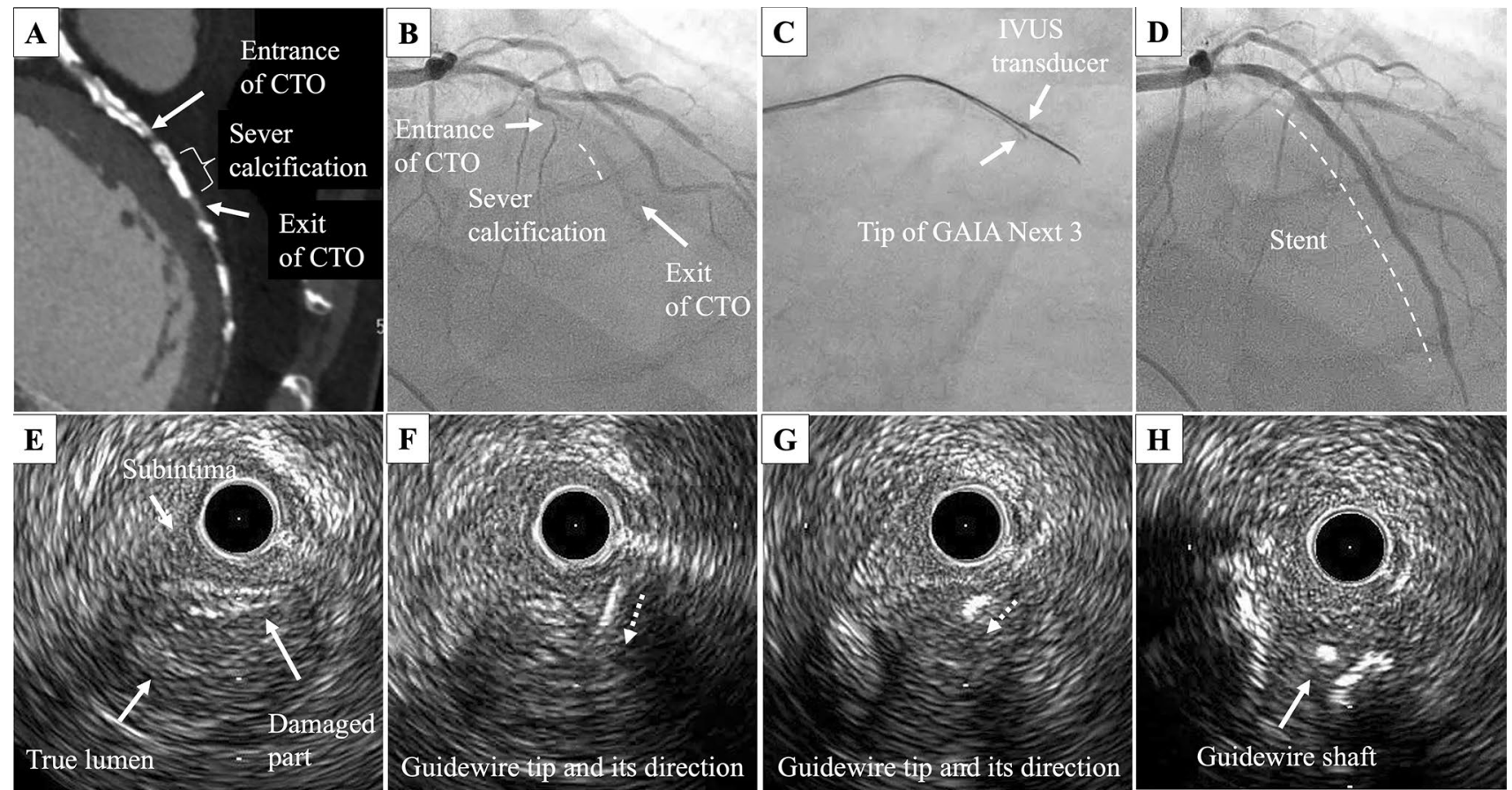

Fig. 1 Computed tomographic image (A), angiographic images (B) prior to, $\mathbf{C}$ during, and $\mathbf{D}$ after the procedure, and intravascular ultrasound images $\mathbf{E}$ prior to, $\mathbf{F}, \mathbf{G}$ during the puncture, and $\mathbf{H}$ after successful puncture. $C T O$ chronic total occlusion

Supplementary Information The online version contains supplementary material available at https://doi.org/10.1007/s12928-022-00846-2.

Open Access This article is licensed under a Creative Commons Attribution 4.0 International License, which permits use, sharing, adaptation, distribution and reproduction in any medium or format, as long as you give appropriate credit to the original author(s) and the source, provide a link to the Creative Commons licence, and indicate if changes were made. The images or other third party material in this article are included in the article's Creative Commons licence, unless indicated otherwise in a credit line to the material. If material is not included in the article's Creative Commons licence and your intended use is not permitted by statutory regulation or exceeds the permitted use, you will need to obtain permission directly from the copyright holder. To view a copy of this licence, visit http://creativecommons.org/licenses/by/4.0/.

\section{Reference}

1. Okamura A, Iwakura K, Iwamoto M, Nagai H, Sumiyoshi A, Tanaka K, et al. Tip detection method using the new IVUS facilitates the 3-dimensional wiring technique for CTO intervention. JACC Cardiovasc Interv. 2020;13:74-82.

Publisher's Note Springer Nature remains neutral with regard to jurisdictional claims in published maps and institutional affiliations. 